\title{
Phosphatidylserine targeting for diagnosis and treatment of human diseases
}

\author{
Kristof Schutters $\cdot$ Chris Reutelingsperger
}

Published online: 4 May 2010

(c) The Author(s) 2010. This article is published with open access at Springerlink.com

\begin{abstract}
Cells are able to execute apoptosis by activating series of specific biochemical reactions. One of the most prominent characteristics of cell death is the externalization of phosphatidylserine (PS), which in healthy cells resides predominantly in the inner leaflet of the plasma membrane. These features have made PS-externalization a well-explored phenomenon to image cell death for diagnostic purposes. In addition, it was demonstrated that under certain conditions viable cells express PS at their surface such as endothelial cells of tumor blood vessels, stressed tumor cells and hypoxic cardiomyocytes. Hence, PS has become a potential target for therapeutic strategies aiming at Targeted Drug Delivery. In this review we highlight the biomarker PS and various PS-binding compounds that have been employed to target PS for diagnostic purposes. We emphasize the $35 \mathrm{kD}$ human protein annexin A5, that has been developed as a Molecular Imaging agent to measure cell death in vitro, and non-invasively in vivo in animal models and in patients with cardiovascular diseases and cancer. Recently focus has shifted from diagnostic towards therapeutic applications employing annexin A5 in strategies to deliver drugs to cells that express PS at their surface.
\end{abstract}

Keywords Apoptosis - Phosphatidylserine ·

Annexin A5 · Molecular Imaging · Targeted Drug Delivery

K. Schutters $(\bowtie) \cdot C$. Reutelingsperger

Department of Biochemistry, Cardiovascular Research Institute

Maastricht, Maastricht University, PO Box 616,

6200 MD Maastricht, The Netherlands

e-mail: k.schutters@maastrichtuniversity.nl

C. Reutelingsperger

e-mail: C.reutelingsperger@maastrichtuniversity.nl

\section{Introduction}

Scientific and technological developments of the past decade have been directed towards the unraveling of molecular fingerprints of distinct diseases in order to facilitate diagnosis and pharmacotherapy by Molecular Imaging (MI) and Targeted Drug Delivery (TDD), respectively. Phosphatidylserine (PS) is one of the molecules that has gained special attention as being part of a cell surface fingerprint of stressed and dying cells. PS is a negatively charged aminophospholipid that is present in all cells and constitutes approximately $2-10 \%$ of total cellular lipid [1]. In addition to a structural function PS is involved in signaling pathways such as protein kinase $\mathrm{C}$ pathways [2] and in localizing intracellular proteins to cytosolic membrane leaflets [3]. PS is normally localized in membrane leaflets that face the cytosol. However, certain conditions can cause translocation of PS to the outer leaflet of the plasma membrane where it may initiate and participate in humoral and cellular processes such as blood coagulation and phagocytosis. Cell surface expression of PS has been found with aging erythrocytes, activated platelets, activated macrophages, endothelial cells of tumor blood vessels, apoptotic cells, apoptotic bodies and cell derived microparticles. It is generally believed that phagocytes in healthy tissues rapidly and efficiently remove PS expressing cells and cell remnants. Diseased tissues on the other hand have a sustained presence of cell surface expressed PS as a result of an imbalance in appearance and clearance of PS expressing cells and cell remnants. Cell surface expressed PS is therefore a useful target for Molecular Imaging and Targeted Drug Delivery (TDD) strategies. This review addresses PS and its binding ligands with potential applications in diagnosis and treatment of a variety of diseases including cardiovascular diseases and cancer. 


\section{PS-asymmetry of the plasma membrane}

PS is ubiquitously present in prokaryotic and eukaryotic cells and constitutes about $2-10 \%$ of total cellular lipids depending on species and cell type. PS synthetic pathways differ between bacteria, yeast and mammals [4]. Mammalian cells synthesize PS predominantly by converting enzymatically phosphatidylcholine (PC) and phosphatidylethanolamine (PE) through a serine exchange reaction. The enzymes PS-synthase 1 (substrate PC) and PS-synthase 2 (substrate PE) catalyze the conversion and are present in the endoplasmatic reticulum (Fig. 1).

PS appears to be crucial to the cell and, as such, is produced by different biosynthetic routes that can compensate each other to maintain a certain minimal level of PS in case one route fails [5, 6]. Cellular PS is non-randomly distributed through several transport mechanisms including vesicular transport and lipid-transfer protein mediated lipid-exchange between juxtapositioned bilayers [7]. Once present in the PM it is subject to the action of the aminophospholipid transporter (APLT) which translocates PS rapidly from the exoplasmic to the cytoplasmic leaflet if PS appears in the exoplasmic leaflet. APLT also translocates PE albeit at a lower rate [8]. The aminophospholipids are thus moved across the bilayer against their gradient and the energy required for translocation is derived from hydrolysis of ATP. APLT appears to be a member of the family of P4 type ATPases, a class of ATPases that mediate ATP-dependently the transbilayer movement of phospholipids [9]. APLT activity is present in erythrocytes, platelets and nucleate cells [10]. In the latter APLT resides in the PM and in trans-Golgi and Golgi derived secretory vesicles. PS asymmetry, once established, is a relatively stable steady state, and APLT activity is required again if disturbances caused by for example membrane fusion processes during endo- and exocytosis occur. It has been shown that inhibition of APLT activity only results in a slow rate of PS exposure [11] indicating that PS asymmetry of the PM is of importance to cell homeostasis.

\section{Cell surface expression of PS}

Certain conditions may induce cells to release their PS asymmetry of the PM. For example aging of erythrocyte, activation of platelets and apoptosis are accompanied by a sustained appearance of PS in the exoplasmic leaflet $[12,13]$. As indicated above, inhibition of APLT is insufficient to cause rapid and sustained cell surface exposure of PS. An additional mechanism is required to achieve a steady state level of PS in the exoplasmic leaflet. Current main hypothesis describes a scramblase activity to be responsible for PS appearance at the cell surface.

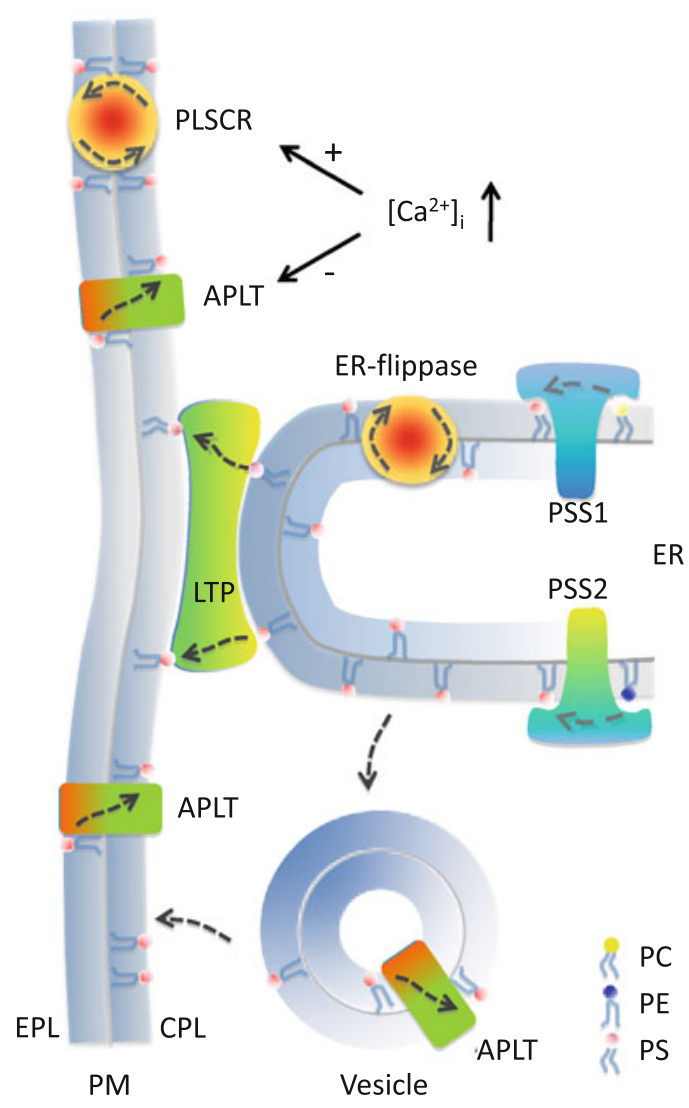

Fig. 1 Schematic presentation of synthesis and transport of PS in mammalian cells. PS is synthesized predominantly by PS-synthase 1 and PS-synthase 2 by converting PC and PE through a serine exchange reaction in the endoplasmic reticulum. After synthesis PS is non-randomly distributed by vesicle transport, membrane fusion and exchange between juxtapositioned bilayers. PS-trafficking pathways are indicated by "dotted arrows". Once present in the PM PS is subject to the action of APLT- and PLSCR-transporters. Increase of cytosolic $\mathrm{Ca}^{2+}$-concentration $\left(\left[\mathrm{Ca}^{2+}\right]_{\mathrm{i}}\right)$ inhibits APLT and activates PLSCR. PSS, PS-synthase; LTP, lipid transfer protein; APLT, aminophospholipid translocase; PLSCR, phosholipid scramblase; ER, endoplasmatic reticulum; EPL, exoplasmic leaflet; CPL, cytoplasmic leaflet; PM, plasma membrane; PC, phosphatidylcholine; PE, phosphatidylethanolamine; PS, phosphatidylserine

Scramblase translocates phospholipids bidirectionally over the two leaflets of the PM thereby collapsing PS asymmetry. Scrambling is rapid, ATP-independent and non-selective for phospholipid species and it causes randomization of the phospholipids over the two membrane leaflets. Scramblase has been demonstrated to operate in erythrocytes [14], activated platelets [15] and apoptotic cells [16]. Several studies have tried to identify the protein(s) that scramble the phospholipids of the PM. Isolation and reconstitution experiments delivered the protein phospholipid scramblase 1 (PLSCR1), which is the most serious candidate up to now [17]. Closer inspection, however, casted doubt because cells were able to scramble PM phospholipids in the absence of PLSCR1 [18] and six 
different cell lines showed a lack of correlation between the level of PLSCR1 expression and the capacity to externalize PS during apoptosis [19]. No other candidates are proposed as yet indicating complexity of phospholipid scrambling and, likely, diversity in scrambling mechanisms. The latter is illustrated by the finding that platelets of a patient with Scott syndrome fail to express PS upon $\mathrm{Ca}^{2+}$-ionophore treatment (a trigger for healthy platelets to expose PS) whilst Scott B-cells normally translocate PS to the cell surface upon execution of apoptosis [20].

Recently an alternative hypothesis was postulated that describes PS externalization as part of membrane repair mechanisms that start to operate during apoptosis and involve fusion of lysosomes with PM [21]. This hypothesis does not require the action of a scramblase protein.

Translocation of PS to the PM exoplasmic leaflet proceeds without compromising the barrier function of the PM. Once in the exoplasmic leaflet PS may participate in a variety of processes depending on type and localization of the PS exposing cell. Circulating erythrocytes for example gradually express PS during aging. PS at the erythrocyte surface functions as an 'eat me' signal towards the reticuloendothelial system, which clears the PS tagged erythrocytes from the circulation by phagocytosis [22]. Platelets can participate in hemostatic and thrombotic processes and while doing so can expose PS at their surface. The PS expressing surface catalyzes coagulation reactions that culminate in the formation of thrombin, which subsequently stabilizes the platelet thrombus by generation of fibrin [23]. Activated macrophages that are engaged to engulf dying cells expose PS at their surface. Inhibition of PS exposure greatly impairs phagocytic capacity of the activated macrophage [24]. Vaccinia virus presents PS at the viral membrane to activate PS dependent macropinocytosis with subsequent infection of the host cell [25]. Macrophages and fibroblasts that are infected with Pichinde Virus express PS at the cell surface [26].

The most important and abundant cellular process that is accompanied by cell surface expression of PS is apoptosis, a biochemically regulated process of cell suicide [27]. Firstly described for apoptotic lymphocytes [13] PS exposure is now appreciated as a ubiquitous phenomenon of apoptosis that is independent of cell type and cell death inducing trigger [28] and that is phylogenetically conserved [29]. PS on the surface of an apoptotic cell is one of the most important 'eat me' flags that not only triggers engulfment but also activates signaling pathways that control cholesterol efflux and expression of anti- and proinflammatory cytokines [30]. In addition, PS on the apoptotic cell surface is involved in regulation of immune response towards antigens of the apoptotic cell [31]. Cells that die by executing a non-apoptotic cell death program also activate a machinery that drives cells surface expression of PS [32] indicating that PS expression is an important phenomenon in dealing with cell death in the context of the multicellular organism. Recognition and engulfment of PS expressing cells are extremely efficient in healthy tissues, which therefore contain, if any, a low steady state level of PS expressing cells. Pathologies can change drastically the balance between appearance and clearance of PS expressing cells towards a sustained presence of PS expressing cells and cell remnants such as apoptotic bodies and cell derived microparticles in diseased tissue. As such surface expressed PS is potentially an informative biomarker for diagnosing disease and evaluating efficacy of therapy. In addition cell surface expressed PS may serve as a target for TDD strategies to deliver therapeutic compounds specifically to diseased tissue.

\section{PS binding ligands}

In order to exploit fully the potential of PS as a target for Molecular Imaging (diagnosis) and TDD (therapy) ligands should be available that bind selectively and with high affinity to cell surface expressed PS in the complexity of the multicellular organism. To date a variety of PS-binding compounds have been reported in the literature including proteins [2], peptides [33-36] and small chemical entities [37]. As Molecular Imaging agents peptides and small chemical compounds generally have the advantage of being quickly and efficiently cleared from the blood circulation. The signal to background ratio is favorably affected by such kinetics. However, the disadvantage of these compounds concerns their low affinity for binding PS. Proteins on the other hand can exhibit higher affinities for PS but proteins are usually cleared from the blood circulation with slower kinetics. The next sections will highlight in particular the proteins annexin A5, synaptotagmin I and lactadherin with which a body of experience has been build about Molecular Imaging of PS expressing cells in vivo in animal models employing a variety of imaging modalities. To date annexin A5 is the only protein that has been used to visualize PS expressing cells in patients using nuclear imaging. Key characteristics of these three proteins are summarized in Table 1.

\section{Annexin A5}

Annexin A5 was discovered as an anticoagulant protein of vascular tissue [38]. It is a non-glycosylated single chain protein that belongs to the annexin super-gene family. Its polypeptide is organized in an N-terminal tail with a $\mathrm{C}$-terminal core containing four domains that form the annexin-core, a slightly bent surface with a convex shape that interacts with the PS containing phospholipid 

Domain I annexin A5

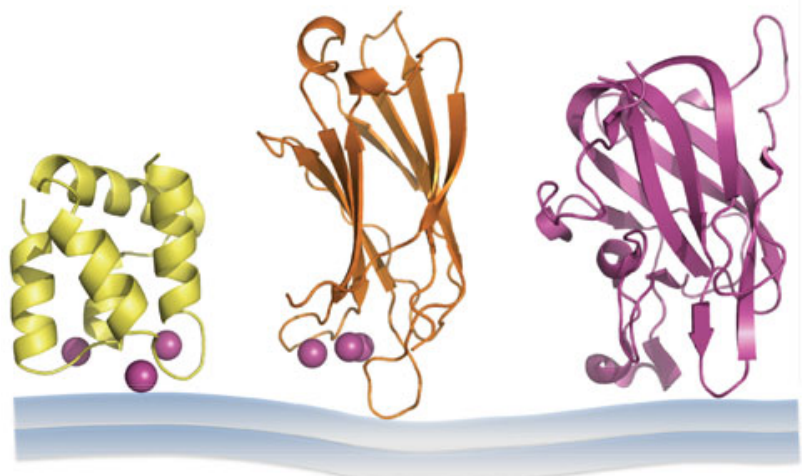

synaptotagmin I C2A-domain

lactadherin C2-domain

PS-containing membrane

Fig. 2 Ribbon presentations of the structures of the PS binding domains of annexin A5, synaptotagmin I and lactadherin. The structural data were retrieved from the Protein Data Bank (PDB) entries 1AVR, 1BYN and 3BN6, respectively. The purple spheres represent $\mathrm{Ca}^{2+}$-ions

membrane $[39,40]$. PS binding of annexin A5 is $\mathrm{Ca}^{2+}$ dependent. $\mathrm{Ca}^{2+}$-ions bind to the annexin core surface at type II $\mathrm{Ca}^{2+}$-binding sites [41] and form the prime contact by coordinating carbonyl and carboxyl groups of the protein and phosphoryl moieties of the glycerol backbone of membrane phospholipids [40]. The domains are composed mainly of $\alpha$-helices and the $\mathrm{Ca}^{2+}$-binding sites protrude as loops (Fig. 2). The overall PS binding affinity arises from a collaboration between the $\mathrm{Ca}^{2+}$-binding sites of the four domains with a dominant role for domain 1 [42]. The complex structure/function relationship of annexin A5 and PS binding has sofar prevented the generation of smaller fragments or mimetics that interact with PS with comparable binding affinity. In solution annexin A5 is present as monomer but once bound to PS-expressing membrane three monomers build a trimer by protein-protein interaction and trimers assemble in a two-dimensional lattice covering the PS expressing surface by trimer-trimer interactions [43]. The two-dimensional protein network of annexin A5 at a PS expressing cell surface drives internalization of annexin A5 [44]. Syntaptotagmin I and lactadherin have reported properties neither of twodimensional crystallisation on the phospholipid surface nor of internalization via the PS portal of cell entry. Annexin A5 binds PS containing membranes with a $\mathrm{K}_{\mathrm{d}}$ in the range of $0.1-2 \mathrm{nM}$ (Table 1).

\section{Synaptotagmin I}

Synaptotagmin I is a synaptic vesicle membrane protein with a short $\mathrm{N}$-terminal intravesicular sequence, a single transmembrane region, and a cytoplasmic region containing two domains with homology to the C2-domain of Protein Kinase C [45-47]. It functions intracellularly as $\mathrm{Ca}^{2+}$-sensor to mediate synaptic vesicle fusion upon rise of cytoplasmic $\mathrm{Ca}^{2+}$-levels. Phospholipid binding of synaptotagmin I is mediated by its C2-domains which bind preferentially the negatively charged phospholipids PS and phosphatidylinositol $[48,49]$. PS binding can be exhibited by a single $\mathrm{C} 2$ domain as was shown for the first $\mathrm{C} 2$ domain $(\mathrm{C} 2 \mathrm{~A})$ that was expressed recombinantly by $E$. coli [37]. The C2A-domain is composed of stable eight-stranded-sandwiches with flexible loops emerging from the top and bottom [48]. These loops bind $\mathrm{Ca}^{2+}$ [50] and acquire subsequently a positive electrostatic potential that becomes attracted by negatively charged phospholipid membranes (Fig. 2) [51]. The C2B domain can bind phospholipids $\mathrm{Ca}^{2+}$-independently [52]. The dissociation constant $\left(\mathrm{K}_{\mathrm{d}}\right)$ of synaptotagmin I binding to PS is within the 15-40 nM-range (Table 1).

\section{Lactadherin}

Lactadherin is a glycosylated protein that was firstly discovered as a component of milk fat globule membranes [53]. It contains an EGF-like domain harbouring an RGD sequence that mediates interaction with the integrin receptors $\alpha_{\mathrm{v}} \beta_{3 / 5}$. At the C-terminal end of the RGD-containing EGF-like domain reside two $\mathrm{C}$ domains bearing homologies with the $\mathrm{C} 1$ and $\mathrm{C} 2$ domains of blood coagulation factors $\mathrm{V}$ and VIII. Lactadherin functions as bridging molecule facilitating phagocytosis of dying cells $[54,55]$. The RGDmotif interacts with integrin receptors on the surface of phagocytes and the $\mathrm{C} 2$-like domain binds in a $\mathrm{Ca}^{2+}$-independent manner with cell surface expressed PS [56]. The C2-domain of lactadherin contains a $\beta$-barrel core with

Table 1 Key characteristics of the PS binding proteins annexin A5, synaptotagmin I and lactadherin

\begin{tabular}{llllll}
\hline Protein & MW $(\mathrm{kD})$ & Source & PS-binding & \\
\cline { 3 - 6 } & & & Domain & $\mathrm{Ca}^{2+}$-required & $\mathrm{K}_{\mathrm{d}}\left(\mathrm{nM}^{2}\right)$ \\
\hline Annexin A5 & 36 & Human, expressed in E. coli [70] & Annexin core & Yes & $0.1-2[108,109]$ \\
Synaptotagmin I & 65 & Human, C2A domain expressed in E. coli [83] & C2A-domain & Yes & 15-40 [82] \\
Lactadherin & 47 & Bovine, purified from milk [110] & C2-domain & No & $3-4[110]$ \\
\hline
\end{tabular}

${ }^{a}$ Despite similar nomenclature, the C2-domains of synaptotagmin I and lactadherin do not share sequence homology 
protruding hydrophobic residues that interact $\mathrm{Ca}^{2+}$-independently with PS (Fig. 2). The C2-domain of lactadherin shows no significant homology with the $\mathrm{C} 2 \mathrm{~A}$ domain of synaptotagmin I. Interestingly lactadherin binds PS in a stereo-specific manner [57]. Stereo-specificity has not been observed for the PS binding proteins synaptotagmin I and annexin A5. Lactadherin binds to PS containing membranes with a $K_{d}$ ranging from 2 to $4 \mathrm{nM}$ (Table 1).

\section{Other PS binding proteins}

Other PS-binding proteins that have potential to be used as ligands for imaging PS expressing cells include $\mathrm{T}$ cell immunoglobin mucins, $\gamma$-carboxyglutamic acid (Gla) containing proteins and antibodies directed against PS. T cell immunoglobulin mucin 1 and 4 (TIM-1, TIM-4), both members of the TIM-family were originally identified as a marker of $\mathrm{T}$ cell subsets. TIM-proteins are transmembrane proteins that share an immunoglobulin variable domain containing 6 cysteines, a mucin like domain, a transmembrane domain and a cytoplasmic domain [58]. Both TIM-1 and TIM-4 act as a phagocyte receptor for PS expressed on the apoptotic cell [59]. The immunoglobulin domain binds specifically to PS with a Kd of approximately $2 \mathrm{nM}$ [60].

Gla-domain containing proteins such as vitamin K-dependent blood coagulation factors bind PS through a $\mathrm{Ca}^{2+}$-mediated interaction between Gla-residues and PS [61]. Gla-domain containing proteins generally bind PS expressing membranes with a $K_{d}$ in the $\mathrm{nM}$ range.

Immunization procedures with PS as antigen may generate antibodies against PS [62]. However, in most cases immunization results in the generation of antibodies that recognize plasma proteins bound to PS. To target PS on tumor vasculature, the murine monoclonal antibody $3 \mathrm{G} 4$ was generated [63]. It appeared that 3G4 does not bind PS directly but through plasma protein 2-glycoprotein 1 that was bound to PS [64]. Plasma protein 2-glycoprotein 1 binds weakly to anionic phospholipids whereas in presence of $3 \mathrm{G} 4$ its affinity for anionic membranes increases significantly.

\section{Molecular Imaging of PS}

As described above PS expressing cells and cell remnants accumulate in diseased tissues predominantly as a result of the activation of cell death processes and insufficient clearance of the PS expressing cells. Apoptosis is the major process of cell death and plays a role in a wide range of pathologies [65-69]. Therefore non-invasive and tomographic imaging of surface expressed PS has gained interest not only in basic and translational research but also in various clinical disciplines to support diagnosis, localize pathological sites and assess efficacy of therapy. The availability of the PS binding ligand annexin A5 has boosted research and development of Molecular Imaging of PS. To date imaging studies in animal models have been carried out predominantly with various labeled forms of annexin A5. A number of papers have reported about the use of a labeled fusion protein of Gluthation-S-transferase (GST) and the C2A domain of synaptotagmin I. Lactadherin is the less employed one of the three PS binding proteins. Its use has been confined to in vitro studies sofar.

\section{Molecular Imaging of PS with Annexin A5}

The recombinantly expressed human annexin A5 exhibits PS binding properties identical to annexin A5 purified from human tissue [70]. Availability of recombinant annexin A5 spurred synthesis of a wide range of labeled forms of annexin A5 to accomodate PS imaging with modalities such as optical, radionuclide and magnetic resonance imaging [71, 72]. Annexin A5 is labeled with reporter compounds through chemical coupling mostly to primary amino groups of annexin A5. Since these are also present on the surface of the annexin core amine-based coupling may compromise the PS binding potency [73, 74]. In order to avoid deleterious effects of coupling, annexin A5 variants have been generated for site-directed labeling at the concave side of the molecule using thiol chemistry. Annexin A5 variants have been generated with thiol-linkage sites in extensions of the N-terminus $[75,76]$ and thiollinkage sites within the $\mathrm{N}$-terminal tail and the concave side of annexin A5 to which small compounds (chelators of radionuclides [77] and fluorochromes [78]) as well as particles with diameters ranging from 10 to $100 \mathrm{~nm}$ (iron oxide nanoparticles [79] and liposomes [80, 81]) have been coupled successfully without impairing PS binding. This so-called 'second generation' annexin A5 has improved biodistribution and PS binding properties as compared to amine-labeled wildype annexin A5.

\section{Molecular Imaging of PS with C2A domain} of Synaptotagmin I

Synaptotagmin I is less suitable as a whole molecule for Molecular Imaging because of its transmembrane domain. The soluble PS binding C2A domain was expressed recombinantly by $E$. coli as a fusion protein with GST. Although the affinity for binding PS is higher for C2A $\left(K_{d}=20-40 \mathrm{nM}\right)$ as compared to the fusion protein C2AGST $\left(\mathrm{K}_{\mathrm{d}}= \pm 115 \mathrm{nM}\right)$ it was decided to develop C2A-GST as a s ligand because labeling of $\mathrm{C} 2 \mathrm{~A}$ interfered with PS binding [82]. Labeling of GST-C2A likely occurred predominantly at the GST moiety. C2A-GST can be conjugated to fluorochromes, radionuclides and superparamagnetic iron oxide particles using random chemical linkage while 
retaining PS binding property $[83,84]$. Whether site-directed chemical linkage will yield a superior PS imaging ligand has not been reported so far.

Molecular Imaging of PS with lactadherin

For PS imaging purposes lactadherin was purified from bovine milk [85]. To date PS imaging with lactadherin has been limited to in vitro studies only. Lactadherin has been coupled to fluorescein isothiocyanate via random chemical linkage to accomodate optical imaging [85]. It is claimed that lactadherin has several advantages as a PS imaging agent over annexin A5 and synaptotagmin I. It binds membranes in a way that is proportional to PS content and independent of both phosphatidylethanolamine and $\mathrm{Ca}^{2+}$ [57]. The latter feature is, however, not a benefit in vivo because ionized extracellular $\mathrm{Ca}^{2+}$ levels fluctuate around $1 \mathrm{mM}$ which is more than sufficient to promote binding of annexin A5 and C2A-GST to PS expressing membranes. The drawback of lactadherin is its posttranslational modification which precludes expression of functional lactadherin recombinantly in an E. coli system.

\section{A 3D FMT Imaging}
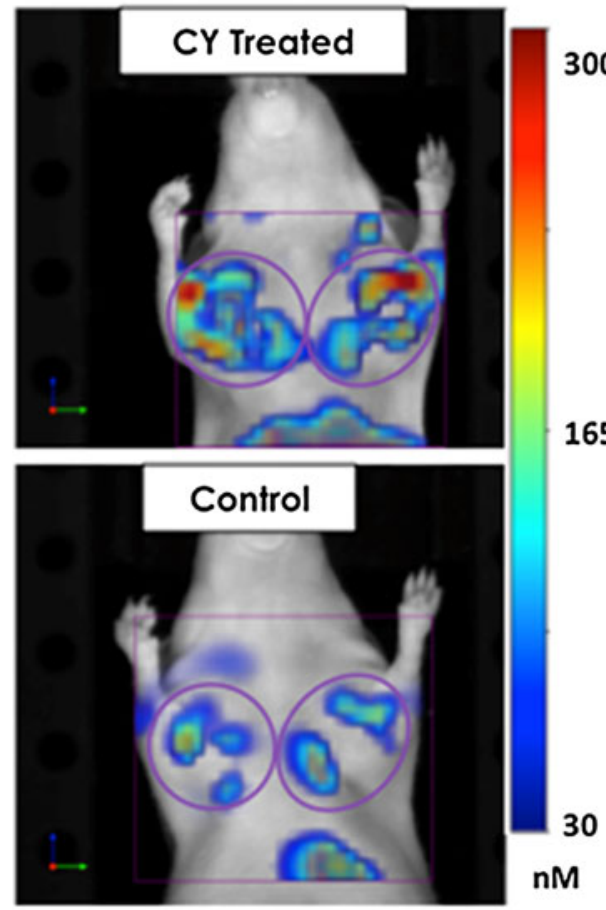

165

Fig. 3 Non-invasive PS-imaging of efficacy of anti-cancer treatment. Mice were implanted with HT-29 human colorectal adenocarcinoma cells in both upper mammary fat pads, and tumors were allowed to grow for 10 days. To assess the acute induction of apoptosis, animals received a single IP injection of cyclophosphamide (CY; $170 \mathrm{mg} / \mathrm{kg}$ ). Animals were injected with second generation annexin A5 coupled to Vivo 750 (annexin-Vivo 750, VisEn Medical) $24 \mathrm{~h}$ post-CY treatment
The general picture of all imaging studies utilizing annexin A5 and C2A-GST depicts feasibility of PS imaging with non-invasive techniques including optical, radionuclide and magnetic resonance imaging. Non-invasive PS imaging can be employed to accomplish various goals including understanding pathogenesis of cardiovascular diseases such as heart failure [86] and atherosclerosis [87, 88] and evaluating therapeutic in vivo efficacy of drugs such as statins [89] and anti-cancer compounds [90, 91]. Most preclinical non-invasive PS imaging has been carried out with radionuclide and magnetic resonance imaging. Low tissue penetration of photons and autofluorescence of extracellular matrix components have been hampering development of non-invasive optical imaging of PS. Recently near-infrared fluorescent (NIRF) probes and fluorescence mediated tomography (FMT) have been developed rendering non-invasive optical imaging feasible [92]. Second-generation annexin A5 has been coupled to the NIRF probe Vivo-750 via thiol-chemistry and employed successfully to quantify the anti-cancer effect of cytotoxic compounds in a mouse cancer model using noninvasive FMT (Fig. 3).

\section{B $\quad$ FMT Quantification}

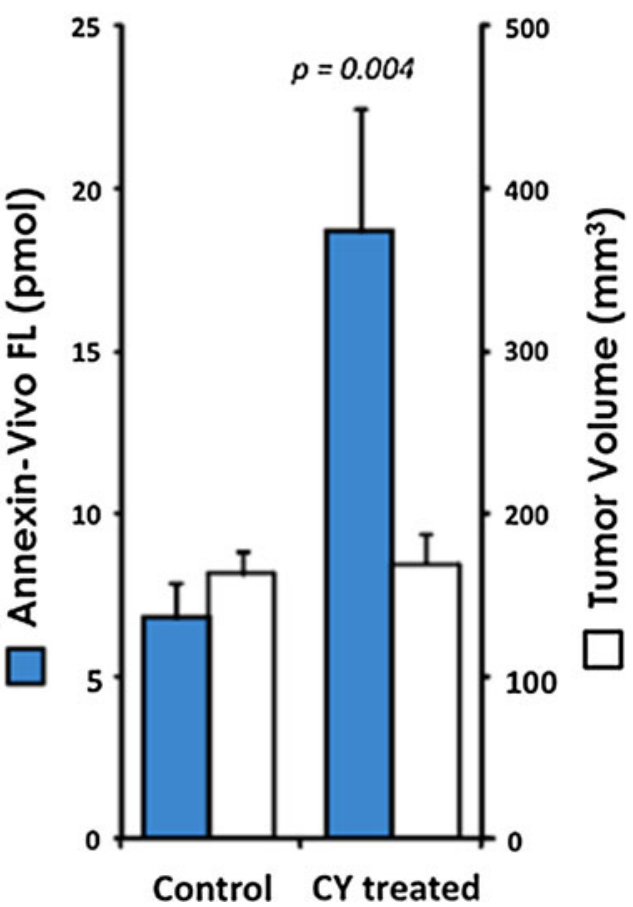

and imaged $2 \mathrm{~h}$ later by FMT 2500 (VisEn Medical) fluorescence tomography. Representative images of treated and untreated HT-29 mice were selected to reflect the mean group tumor fluorescence intensities. (a) 3D FMT images were acquired, and (b) the effects on tumor fluorescence and volume were assessed. Results indicate anticancer efficacy of CY in this model. Data and images courtesy of VisEn Medical Inc., Bedford, MA, USA 
Table 2 Overview of preclinical and clinical imaging of PS for a variety of diseases and diagnostic purposes

\begin{tabular}{llll}
\hline Medical field & Diagnostic purpose of PS imaging & \multicolumn{2}{l}{ References } \\
\cline { 2 - 3 } & & Preclinical studies & Clinical studies \\
\hline Oncology & Early assessment of efficacy of therapy & {$[90,91,111-114]$} \\
& Prognosis of survival & \\
Cardiology & Early diagnosis of heart failure & {$[66,86,120-123]$} \\
& Early assessment of cardiac toxicity & {$[124]$} & {$[125-127]$} \\
Vascular medicine & Assessment of cardiac ischemia/reperfusion injury & {$[128,129]$} \\
Orthopaedics & Identification of unstable atherosclerotic plaque & \\
Organ transplantation & Assessment of infection of prostheses & {$[132]$} \\
Gastroenterology & Allograft rejection & {$[105]$} \\
Neurology & Assessment of efficacy of therapy in Crohn's disease & {$[135,136]$} \\
Ophthalmology & Identifying regions of cerebral injury & {$[139,140]$} \\
Autoimmune diseases & Assessment of retinal neurodegeneration & {$[141]$} \\
Metabolic diseases & Identifying regions of rheumatoid arthritis & {$[142]$} \\
\hline
\end{tabular}

PS imaging has entered the process of translation from preclinical settings to the clinical arena. Availability of clinical grade recombinant human annexin A5 for labeling with Technetium $\left(\mathrm{Tc}^{99 \mathrm{~m}}\right)$ and Single Photon Emission Tomography (SPECT) has catalyzed clinical studies with PS imaging in various patients to assess the significance of PS imaging for diagnosis and rapid evaluation of efficacy of therapy. Table 2 summarizes medical fields in which Molecular Imaging of PS has been interrogated in preclinical models and in patients. These studies show promise for non-invasive imaging of PS to catalyze drug development in preclinical settings and to support diagnosis and evaluation efficacy of treatment shortly after start of therapy in the clinic.

\section{PS as target for Targeted Drug Delivery (TDD) strategies}

TTD is a strategy the goal of which is to treat disease effectively with minimal detrimental side-effects. Such strategies are especially of importance to treatments in which toxic substances are needed to combat disease. TDD is based on the principle of Paul Ehrlich's 'Magic Bullet' which in fact is a therapeutic compound that is guided to the diseased lesion by a targeting function. The targeting function can be an integral part of the therapeutic compound or can be deliberately attached to the drug [93]. Cell surface expressed PS is potentially an attractive target for TDD considering the body of experience with PS as a biomarker for Molecular Imaging [93, 94].

A wide range of diseases may benefit from PS based TDD strategies (see Table 2). In general PS is expressed by dying and dead cells and cell remnants that accumulate in diseased lesions such as atherosclerotic plaques and tumours. In such lesions PS may function to accumulate PS seeking 'Magic Bullets' and their therapeutic cargo, which may for example encompass enzymes or cytotoxic substances. Recent experiments indicated that cells, which are not committed to execute cell death, may also express PS on their surface under specific conditions. Endothelial cells of tumour vasculature for example express PS while being alive [95]. Cardiomyocytes that have been submitted to brief ischemia express PS before the apoptotic machinery trespasses the point of no return [96]. These cells can be targeted with therapeutic substances that either kill (tumour endothelial cells) or rescue (stressed cardiomyocytes) the PS expressing cell. Efficient intracellular delivery of the therapeutic substance is then necessary. Annexin A5 has been shown to be internalized into the PS expressing cell as a consequence of its property to form a 2-dimensional lattice on the cell surface [44]. C2A-domain of synaptotagmin I and lactadherin have no reported property of internalization into PS expressing cells.

Recent reports underscored feasibility and applicability of the concept of PS targeting in TDD strategies. Annexin A5 was used as vector to target coagulation and fibrinolytic enzymes to sites of PS expressing cells in the vasculature [97-99]. These PS seeking 'Magic bullets' were constructed by molecular fusion [97, 99] or chemical coupling [98] of annexin A5 and the enzyme. Annexin A5 by itself integrates PS targeting and therapeutic function because once bound to PS it blocks the inflammatory and immunomodulatory activities of surface expressed PS [100]. Recently a homodimer of annexin A5 (diannexin) was constructed with the purpose to prolong the blood 
circulation time and, hence, to increase therapeutic efficacy of annexin A5 to attenuate ischemia/reperfusion induced injury of organs [101].

PS has also been targeted with the antibody 3G4 (bavituximab), which binds with high affinity to complexes of $\beta$-2-glycoprotein I and PS. 3G4 was used therapeutically as adjuvant therapy in viral infections in which PS surface expression is essential to successful infection [26] and in mouse models of cancer, which have tumour vasculature with PS expressing endothelial cells [102, 103].

Diannexin and bavituximab are the first PS-targeting agents that have entered clinical trials to demonstrate therapeutic activity in patients with kidney transplants, chronic hepatic $\mathrm{C}$ virus and HIV and cancer (http:// clinicaltrials.gov).

\section{Conclusion and future perspectives}

PS is one of the most prominent and ubiquitous fingerprints of cells in diseased tissues and, therefore, an attractive target for Molecular Imaging and translation into clinical applications. Its ubiquity has both advantages and disadvantages. On the one hand a broad spectrum of diseases can be imaged with a single compound but on the other hand ubiquity is accompanied by reduced specificity requiring additional diagnostic steps in order to differentiate. This is exemplified by reports that show that annexin A5 accumulation in the heart of a patient can be the result of acute myocardial infarction [104], ongoing heart failure [105], an intracardiac tumour [106] and infection [107]. Specificity can be increased by more accurate anatomic mapping of the sites where annexin A5 accumulates for example by combining imaging technologies such as SPECT/CT and PET/CT. Development of imaging technology goes in the direction of pin-pointing location of radioisotopes in the human body. More accurate localisation of PS expressing cells will increase the value of PS as a diagnostic biomarker.

PS ubiquity clearly affects TDD aiming at PS. As with Molecular Imaging ubiquity has the advantage of broad application of the PS-TDD concept. Differentiation can be accomplished by tuning the therapeutic cargo of the PS seeking 'Magic Bullet' for a specific disease. The disadvantage potentially resides within the side-effects of treatment. No clear picture exists as yet about therapeutic efficacy in relation to undesired side-effects of PS seeking 'Magic Bullets'. Future studies are necessary in order to fully appreciate the value of PS as a target for TDD strategies to treat diseases.

Acknowledgements Part of this work was financially supported by the European Union through the grant Euregional PACT II by the Interreg IV program of Grensregio Vlaanderen-Nederland (IVA-
VLANED-1.20). The authors are indebted to Dr. Gerry Nicolaes (Dept. Biochemistry, Molecular Modeling and Structure Analysis, Maastricht University) for preparing Fig. 2 with the PS binding domain structures.

Open Access This article is distributed under the terms of the Creative Commons Attribution Noncommercial License which permits any noncommercial use, distribution, and reproduction in any medium, provided the original author(s) and source are credited.

\section{References}

1. Vance JE, Steenbergen R (2005) Metabolism and functions of phosphatidylserine. Prog Lipid Res 44(4):207-234

2. Stace CL, Ktistakis NT (2006) Phosphatidic acid- and phosphatidylserine-binding proteins. Biochim Biophys Acta 1761(8): 913-926

3. Yeung $\mathrm{T}$ et al (2008) Membrane phosphatidylserine regulates surface charge and protein localization. Science 319(5860):210 213

4. Yamaji-Hasegawa A, Tsujimoto M (2006) Asymmetric distribution of phospholipids in biomembranes. Biol Pharm Bull 29(8):1547-1553

5. Bergo MO et al (2002) Defining the importance of phosphatidylserine synthase 2 in mice. J Biol Chem 277(49):4770147708

6. Arikketh D, Nelson R, Vance JE (2008) Defining the importance of phosphatidylserine synthase-1 (PSS1): unexpected viability of PSS1-deficient mice. J Biol Chem 283(19):12888-12897

7. Holthuis JC, Levine TP (2005) Lipid traffic: floppy drives and a superhighway. Nat Rev Mol Cell Biol 6(3):209-220

8. Morrot G et al (1989) Aminophospholipid translocase of human erythrocytes: phospholipid substrate specificity and effect of cholesterol. Biochemistry 28(8):3456-3462

9. Puts CF, Holthuis JC (2009) Mechanism and significance of P4 ATPase-catalyzed lipid transport: lessons from a $\mathrm{Na}+/ \mathrm{K}+-$ pump. Biochim Biophys Acta 1791(7):603-611

10. Devaux PF (1992) Protein involvement in transmembrane lipid asymmetry. Annu Rev Biophys Biomol Struct 21:417-439

11. Bitbol $\mathrm{M}$ et al (1987) Ion regulation of phosphatidylserine and phosphatidylethanolamine outside-inside translocation in human erythrocytes. Biochim Biophys Acta 904(2):268-282

12. Zwaal RF, Schroit AJ (1997) Pathophysiologic implications of membrane phospholipid asymmetry in blood cells. Blood 89(4):1121-1132

13. Fadok VA et al (1992) Exposure of phosphatidylserine on the surface of apoptotic lymphocytes triggers specific recognition and removal by macrophages. J Immunol 148(7):2207-2216

14. Basse $F$ et al (1996) Isolation of an erythrocyte membrane protein that mediates $\mathrm{Ca}^{2+}$-dependent transbilayer movement of phospholipid. J Biol Chem 271(29):17205-17210

15. Williamson $P$ et al (1995) Continuous analysis of the mechanism of activated transbilayer lipid movement in platelets. Biochemistry 34(33):10448-10455

16. Verhoven B, Schlegel RA, Williamson P (1995) Mechanisms of phosphatidylserine exposure, a phagocyte recognition signal, on apoptotic T lymphocytes. J Exp Med 182(5):1597-1601

17. Zhou Q et al (1997) Molecular cloning of human plasma membrane phospholipid scramblase. A protein mediating transbilayer movement of plasma membrane phospholipids. J Biol Chem 272(29):18240-18244

18. Zhou Q et al (2002) Normal hemostasis but defective hematopoietic response to growth factors in mice deficient in phospholipid scramblase 1. Blood 99(11):4030-4038 
19. Zhou Q et al (2000) Transcriptional control of the human plasma membrane phospholipid scramblase 1 gene is mediated by interferon-alpha. Blood 95(8):2593-2599

20. Williamson P et al (2001) Phospholipid scramblase activation pathways in lymphocytes. Biochemistry 40(27):8065-8072

21. Mirnikjoo B, Balasubramanian K, Schroit AJ (2009) Suicidal membrane repair regulates phosphatidylserine externalization during apoptosis. J Biol Chem 284(34):22512-22516

22. Allen TM, Williamson P, Schlegel RA (1988) Phosphatidylserine as a determinant of reticuloendothelial recognition of liposome models of the erythrocyte surface. Proc Natl Acad Sci USA 85(21):8067-8071

23. Heemskerk JW, Bevers EM, Lindhout T (2002) Platelet activation and blood coagulation. Thromb Haemost 88(2):186-193

24. Hamon $\mathrm{Y}$ et al (2000) ABC1 promotes engulfment of apoptotic cells and transbilayer redistribution of phosphatidylserine. Nat Cell Biol 2(7):399-406

25. Mercer J, Helenius A (2008) Vaccinia virus uses macropinocytosis and apoptotic mimicry to enter host cells. Science 320(5875):531-535

26. Soares MM, King SW, Thorpe PE (2008) Targeting inside-out phosphatidylserine as a therapeutic strategy for viral diseases. Nat Med 14(12):1357-1362

27. Hengartner MO (2000) The biochemistry of apoptosis. Nature 407(6805):770-776

28. Martin SJ et al (1996) Phosphatidylserine externalization during CD95-induced apoptosis of cells and cytoplasts requires ICE/ CED-3 protease activity. J Biol Chem 271(46):28753-28756

29. van den Eijnde SM et al (1998) Cell surface exposure of phosphatidylserine during apoptosis is phylogenetically conserved. Apoptosis 3(1):9-16

30. Ravichandran KS, Lorenz U (2007) Engulfment of apoptotic cells: signals for a good meal. Nat Rev Immunol 7(12):964-974

31. Chaurio RA et al (2009) Phospholipids: key players in apoptosis and immune regulation. Molecules 14(12):4892-4914

32. Leist M, Jaattela M (2001) Four deaths and a funeral: from caspases to alternative mechanisms. Nat Rev Mol Cell Biol 2(8):589-598

33. Burtea C et al (2009) Peptidic targeting of phosphatidylserine for the MRI detection of apoptosis in atherosclerotic plaques. Mol Pharm 6(6):1903-1919

34. Igarashi $\mathrm{K}$ et al (1995) A novel phosphatidylserine-binding peptide motif defined by an anti-idiotypic monoclonal antibody. Localization of phosphatidylserine-specific binding sites on protein kinase $\mathrm{C}$ and phosphatidylserine decarboxylase. J Biol Chem 270(49):29075-29078

35. Laumonier $\mathrm{C}$ et al (2006) A new peptidic vector for molecular imaging of apoptosis, identified by phage display technology. $\mathrm{J}$ Biomol Screen 11(5):537-545

36. Thapa $\mathrm{N}$ et al (2008) Discovery of a phosphatidylserine recognizing peptide and its utility in molecular imaging of tumor apoptosis. J Cell Mol Med 12(5A):1649-1660

37. Hanshaw RG, Smith BD (2005) New reagents for phosphatidylserine recognition and detection of apoptosis. Bioorg Med Chem 13(17):5035-5042

38. Reutelingsperger CP, Hornstra G, Hemker HC (1985) Isolation and partial purification of a novel anticoagulant from arteries of human umbilical cord. Eur J Biochem 151(3):625-629

39. Huber R, Romisch J, Paques EP (1990) The crystal and molecular structure of human annexin $\mathrm{V}$, an anticoagulant protein that binds to calcium and membranes. EMBO J 9(12):38673874

40. Huber $\mathrm{R}$ et al (1990) The calcium binding sites in human annexin $\mathrm{V}$ by crystal structure analysis at 2.0 A resolution. Implications for membrane binding and calcium channel activity. FEBS Lett 275(1-2):15-21
41. Swairjo MA et al (1995) $\mathrm{Ca}(2+)$-bridging mechanism and phospholipid head group recognition in the membrane-binding protein annexin V. Nat Struct Biol 2(11):968-974

42. Montaville P et al (2002) A new consensus sequence for phosphatidylserine recognition by annexins. J Biol Chem 277(27):24684-24693

43. Oling F, Bergsma-Schutter W, Brisson A (2001) Trimers, dimers of trimers, and trimers of trimers are common building blocks of annexin a5 two-dimensional crystals. J Struct Biol 133(1):55-63

44. Kenis H et al (2004) Cell surface-expressed phosphatidylserine and annexin A5 open a novel portal of cell entry. J Biol Chem 279(50):52623-52629

45. Perin MS et al (1991) Structural and functional conservation of synaptotagmin (p65) in Drosophila and humans. J Biol Chem 266(1):615-622

46. Perin MS et al (1990) Phospholipid binding by a synaptic vesicle protein homologous to the regulatory region of protein kinase $\mathrm{C}$. Nature 345(6272):260-263

47. Perin MS et al (1991) Domain structure of synaptotagmin (p65). J Biol Chem 266(1):623-629

48. Sutton RB et al (1995) Structure of the first C2 domain of synaptotagmin I: a novel $\mathrm{Ca}^{2+} /$ phospholipid-binding fold. Cell 80(6):929-938

49. Zhang X, Rizo J, Sudhof TC (1998) Mechanism of phospholipid binding by the C2A-domain of synaptotagmin I. Biochemistry 37(36): 12395-12403

50. Rizo J, Sudhof TC (1998) C2-domains, structure and function of a universal $\mathrm{Ca}^{2+}$-binding domain. J Biol Chem 273(26):15879_ 15882

51. Murray D, Honig B (2002) Electrostatic control of the membrane targeting of C2 domains. Mol Cell 9(1):145-154

52. Kuo W et al (2009) The calcium-dependent and calcium-independent membrane binding of synaptotagmin 1: two modes of C2B binding. J Mol Biol 387(2):284-294

53. Peterson JA, Patton S, Hamosh M (1998) Glycoproteins of the human milk fat globule in the protection of the breast-fed infant against infections. Biol Neonate 74(2):143-162

54. Hvarregaard J et al (1996) Characterization of glycoprotein PAS-6/7 from membranes of bovine milk fat globules. Eur J Biochem 240(3):628-636

55. Andersen MH et al (1997) Bovine PAS-6/7 binds alpha v beta 5 integrins and anionic phospholipids through two domains. Biochemistry 36(18):5441-5446

56. Andersen MH et al (2000) Functional analyses of two cellular binding domains of bovine lactadherin. Biochemistry 39(20): 6200-6206

57. Shi J, Gilbert GE (2003) Lactadherin inhibits enzyme complexes of blood coagulation by competing for phospholipid-binding sites. Blood 101(7):2628-2636

58. Savill J, Gregory C (2007) Apoptotic PS to phagocyte TIM-4: eat me. Immunity $27(6): 830-832$

59. Kobayashi N et al (2007) TIM-1 and TIM-4 glycoproteins bind phosphatidylserine and mediate uptake of apoptotic cells. Immunity 27(6):927-940

60. Miyanishi M et al (2007) Identification of Tim4 as a phosphatidylserine receptor. Nature 450(7168):435-439

61. Huang $M$ et al (2003) Structural basis of membrane binding by Gla domains of vitamin K-dependent proteins. Nat Struct Biol 10(9):751-756

62. Umeda M et al (1989) Effective production of monoclonal antibodies against phosphatidylserine: stereo-specific recognition of phosphatidylserine by monoclonal antibody. J Immunol 143(7):2273-2279

63. Ran S et al (2005) Antitumor effects of a monoclonal antibody that binds anionic phospholipids on the surface of tumor blood vessels in mice. Clin Cancer Res 11(4):1551-1562 
64. Luster TA et al (2006) Plasma protein beta-2-glycoprotein 1 mediates interaction between the anti-tumor monoclonal antibody $3 \mathrm{G} 4$ and anionic phospholipids on endothelial cells. J Biol Chem 281(40):29863-29871

65. Brown JM, Attardi LD (2005) The role of apoptosis in cancer development and treatment response. Nat Rev Cancer 5(3):231237

66. Yaoita $\mathrm{H}$ et al (2000) Apoptosis in relevant clinical situations: contribution of apoptosis in myocardial infarction. Cardiovasc Res 45(3):630-641

67. Kolodgie FD et al (1999) Apoptosis in human atherosclerotic plaques. Apoptosis 4(1):5-10

68. Narula $J$ et al (1999) Apoptosis in heart failure: release of cytochrome $\mathrm{c}$ from mitochondria and activation of caspase-3 in human cardiomyopathy. Proc Natl Acad Sci USA 96(14):81448149

69. Mattson MP (2000) Apoptosis in neurodegenerative disorders. Nat Rev Mol Cell Biol 1(2):120-129

70. Maurer-Fogy I et al (1988) Cloning and expression of cDNA for human vascular anticoagulant, a $\mathrm{Ca}^{2+}$-dependent phospholipidbinding protein. Eur J Biochem 174(4):585-592

71. Lahorte CM et al (2004) Apoptosis-detecting radioligands: current state of the art and future perspectives. Eur J Nucl Med Mol Imaging 31(6):887-919

72. Faust A et al (2009) Molecular imaging of apoptosis in vivo with scintigraphic and optical biomarkers-a status report. Anticancer Agents Med Chem 9(9):968-985

73. Schellenberger EA, Weissleder R, Josephson L (2004) Optimal modification of annexin $\mathrm{V}$ with fluorescent dyes. Chembiochem 5(3):271-274

74. Tait JF et al (2006) Improved detection of cell death in vivo with annexin $\mathrm{v}$ radiolabeled by site-specific methods. J Nucl Med 47(9):1546-1553

75. Tait JF et al (2000) Development and characterization of annexin V mutants with endogenous chelation sites for $(99 \mathrm{~m}) \mathrm{Tc}$. Bioconjug Chem 11(6):918-925

76. Li X et al (2008) Site-specific labeling of annexin V with F-18 for apoptosis imaging. Bioconjug Chem 19(8):1684-1688

77. De Saint-Hubert M et al (2010) Site-specific labeling of 'second generation' annexin $\mathrm{V}$ with $99 \mathrm{mTc}(\mathrm{CO}) 3$ for improved imaging of apoptosis in vivo. Bioorg Med Chem 18(3):1356-1363

78. Prinzen L et al (2007) Optical and magnetic resonance imaging of cell death and platelet activation using annexin A5-functionalized quantum dots. Nano Lett 7(1):93-100

79. Schellenberger E et al (2008) Linking proteins with anionic nanoparticles via protamine: ultrasmall protein-coupled probes for magnetic resonance imaging of apoptosis. Small 4(2):225230

80. van Tilborg GA et al (2006) Annexin A5-functionalized bimodal lipid-based contrast agents for the detection of apoptosis. Bioconjug Chem 17(3):741-749

81. Garnier B et al (2009) Annexin A5-functionalized liposomes for targeting phosphatidylserine-exposing membranes. Bioconjug Chem 20(11):2114-2122

82. Jung HI et al (2004) Detection of apoptosis using the C2A domain of synaptotagmin I. Bioconjug Chem 15(5):983-987

83. Zhao M et al (2001) Non-invasive detection of apoptosis using magnetic resonance imaging and a targeted contrast agent. Nat Med 7(11):1241-1244

84. Zhao $\mathrm{M}$ et al (2006) 99mTc-labeled C2A domain of synaptotagmin I as a target-specific molecular probe for noninvasive imaging of acute myocardial infarction. J Nucl Med 47(8):13671374

85. Shi J et al (2006) Lactadherin detects early phosphatidylserine exposure on immortalized leukemia cells undergoing programmed cell death. Cytometry A 69(12):1193-1201
86. Sosnovik DE et al (2009) Molecular MRI detects low levels of cardiomyocyte apoptosis in a transgenic model of chronic heart failure. Circ Cardiovasc Imaging 2(6):468-475

87. Isobe $S$ et al (2006) Noninvasive imaging of atherosclerotic lesions in apolipoprotein E-deficient and low-density-lipoprotein receptor-deficient mice with annexin A5. J Nucl Med 47(9):1497-1505

88. Haider $\mathrm{N}$ et al (2009) Dual molecular imaging for targeting metalloproteinase activity and apoptosis in atherosclerosis: molecular imaging facilitates understanding of pathogenesis. J Nucl Cardiol 16(5):753-762

89. Hartung D et al (2005) Resolution of apoptosis in atherosclerotic plaque by dietary modification and statin therapy. J Nucl Med 46(12):2051-2056

90. Wang $\mathrm{F}$ et al (2008) Imaging paclitaxel (chemotherapy)-induced tumor apoptosis with $99 \mathrm{mTc} \mathrm{C} 2 \mathrm{~A}$, a domain of synaptotagmin I: a preliminary study. Nucl Med Biol 35(3):359-364

91. Cornelissen B et al (2005) In vivo apoptosis detection with radioiodinated Annexin V in LoVo tumour-bearing mice following Tipifarnib (Zarnestra, R115777) farnesyltransferase inhibitor therapy. Nucl Med Biol 32(3):233-239

92. Weissleder R, Pittet MJ (2008) Imaging in the era of molecular oncology. Nature 452(7187):580-589

93. Kenis H, Reutelingsperger C (2009) Targeting phosphatidylserine in anti-cancer therapy. Curr Pharm Des 15(23):2719 2723

94. Kenis H, Hofstra L, Reutelingsperger CP (2007) Annexin A5: shifting from a diagnostic towards a therapeutic realm. Cell Mol Life Sci 64(22):2859-2862

95. Ran S, Thorpe PE (2002) Phosphatidylserine is a marker of tumor vasculature and a potential target for cancer imaging and therapy. Int J Radiat Oncol Biol Phys 54(5):1479-1484

96. Kenis $\mathrm{H}$ et al (2010) Annexin a5 uptake in ischemic myocardium: demonstration of reversible phosphatidylserine externalization and feasibility of radionuclide imaging. J Nucl Med 51(2):259-267

97. Tait JF et al (1995) Prourokinase-annexin V chimeras. Construction, expression, and characterization of recombinant proteins. J Biol Chem 270(37):21594-21599

98. Tanaka $\mathrm{K}$ et al (1996) Preparation and characterization of a disulfide-linked bioconjugate of annexin $\mathrm{V}$ with the B-chain of urokinase: an improved fibrinolytic agent targeted to phospholipid-containing thrombi. Biochemistry 35(3):922-929

99. Huang $X$ et al (2006) A soluble tissue factor-annexin V chimeric protein has both procoagulant and anticoagulant properties. Blood 107(3):980-986

100. van Genderen $\mathrm{HO}$ et al (2008) Extracellular annexin A5: functions of phosphatidylserine-binding and two-dimensional crystallization. Biochim Biophys Acta 1783(6):953-963

101. Kuypers FA et al (2007) Interaction of an annexin V homodimer (Diannexin) with phosphatidylserine on cell surfaces and consequent antithrombotic activity. Thromb Haemost 97(3):478-486

102. Huang X, Bennett M, Thorpe PE (2005) A monoclonal antibody that binds anionic phospholipids on tumor blood vessels enhances the antitumor effect of docetaxel on human breast tumors in mice. Cancer Res 65(10):4408-4416

103. He J, Luster TA, Thorpe PE (2007) Radiation-enhanced vascular targeting of human lung cancers in mice with a monoclonal antibody that binds anionic phospholipids. Clin Cancer Res 13(17):5211-5218

104. Hofstra L et al (2000) Visualisation of cell death in vivo in patients with acute myocardial infarction. Lancet 356(9225): 209-212

105. Kietselaer BL et al (2007) Noninvasive detection of programmed cell loss with $99 \mathrm{mTc}$-labeled annexin A5 in heart failure. J Nucl Med 48(4):562-567 
106. Hofstra L et al (2001) In vivo detection of apoptosis in an intracardiac tumor. JAMA 285(14):1841-1842

107. Kietselaer BL, Narula J, Hofstra L (2007) The Annexin code: revealing endocarditis. Eur Heart J 28(8):948

108. Andree HA et al (1990) Binding of vascular anticoagulant alpha (VAC alpha) to planar phospholipid bilayers. J Biol Chem 265(9):4923-4928

109. Tait JF, Gibson D, Fujikawa K (1989) Phospholipid binding properties of human placental anticoagulant protein-I, a member of the lipocortin family. J Biol Chem 264(14):7944-7949

110. Shi J et al (2004) Lactadherin binds selectively to membranes containing phosphatidyl-L-serine and increased curvature. Biochim Biophys Acta 1667(1):82-90

111. Kuge $\mathrm{Y}$ et al (2004) Feasibility of $99 \mathrm{mTc}$-annexin $\mathrm{V}$ for repetitive detection of apoptotic tumor response to chemotherapy: an experimental study using a rat tumor model. J Nucl Med 45(2):309-312

112. Krishnan AS et al (2008) Detection of cell death in tumors by using MR imaging and a gadolinium-based targeted contrast agent. Radiology 246(3):854-862

113. Manning HC et al (2008) Molecular imaging of therapeutic response to epidermal growth factor receptor blockade in colorectal cancer. Clin Cancer Res 14(22):7413-7422

114. Shah $C$ et al (2009) Imaging biomarkers predict response to antiHER2 (ErbB2) therapy in preclinical models of breast cancer. Clin Cancer Res 15(14):4712-4721

115. Kartachova MS et al (2008) 99mTc-HYNIC-rh-annexin-V scintigraphy: visual and quantitative evaluation of early treatment-induced apoptosis to predict treatment outcome. Nucl Med Commun 29(1):39-44

116. Rottey S et al (2007) (99m)Tc-HYNIC Annexin-V imaging of tumors and its relationship to response to radiotherapy and/or chemotherapy. Q J Nucl Med Mol Imaging 51(2):182-188

117. Kurihara $\mathrm{H}$ et al (2008) Imaging and dosimetry of $99 \mathrm{mTc}$ EC annexin V: preliminary clinical study targeting apoptosis in breast tumors. Appl Radiat Isot 66(9):1175-1182

118. Haas RL et al (2004) In vivo imaging of radiation-induced apoptosis in follicular lymphoma patients. Int $\mathbf{J}$ Radiat Oncol Biol Phys 59(3):782-787

119. Loose D et al (2007) Prognostic value of (99m)Tc-HYNIC Annexin-V imaging in squamous cell carcinoma of the head and neck. Eur J Nucl Med Mol Imaging 35(1):47-52

120. Narula $J$ et al (2001) Annexin- $V$ imaging for noninvasive detection of cardiac allograft rejection. Nat Med 7(12):13471352

121. Martinet W, Kockx MM (2001) Apoptosis in atherosclerosis: focus on oxidized lipids and inflammation. Curr Opin Lipidol 12(5):535-541

122. Rossig L, Dimmeler S, Zeiher AM (2001) Apoptosis in the vascular wall and atherosclerosis. Basic Res Cardiol 96(1):1122

123. McCarthy NJ, Bennett MR (2000) The regulation of vascular smooth muscle cell apoptosis. Cardiovasc Res 45(3):747-755

124. Gabrielson KL et al (2008) Detection of dose response in chronic doxorubicin-mediated cell death with cardiac technetium 99m annexin V single-photon emission computed tomography. Mol Imaging 7(3):132-138
125. Zhu X et al (2008) Early uptake of $99 \mathrm{mTc}-\mathrm{C} 2 \mathrm{~A}$ in the acute phase of myocardial infarction as a prognostic indicator for follow-up cardiac dysfunction. Nucl Med Commun 29(9):764769

126. Dumont EA et al (2001) Real-time imaging of apoptotic cellmembrane changes at the single-cell level in the beating murine heart. Nat Med 7(12):1352-1355

127. Fang W et al (2007) SPECT imaging of myocardial infarction using $99 \mathrm{mTc}$-labeled $\mathrm{C} 2 \mathrm{~A}$ domain of synaptotagmin $\mathrm{I}$ in a porcine ischemia-reperfusion model. Nucl Med Biol 34(8):917-923

128. Johnson LL et al (2005) 99mTc-Annexin V imaging for in vivo detection of atherosclerotic lesions in porcine coronary arteries. J Nucl Med 46(7):1186-1193

129. Kolodgie FD et al (2003) Targeting of apoptotic macrophages and experimental atheroma with radiolabeled annexin $\mathrm{V}$ : a technique with potential for noninvasive imaging of vulnerable plaque. Circulation 108(25):3134-3139

130. Kietselaer BL et al (2004) Noninvasive detection of plaque instability with use of radiolabeled annexin A5 in patients with carotid-artery atherosclerosis. N Engl J Med 350(14):1472-1473

131. Lorberboym $\mathrm{M}$ et al (2009) The use of $99 \mathrm{mTc}$-recombinant human annexin $\mathrm{V}$ imaging for differential diagnosis of aseptic loosening and low-grade infection in hip and knee prostheses. $\mathrm{J}$ Nucl Med 50(4):534-537

132. Blankenberg FG et al (2000) Radionuclide imaging of acute lung transplant rejection with annexin V. Chest 117(3):834-840

133. Kown $\mathrm{MH}$ et al (2001) In vivo imaging of acute cardiac rejection in human patients using $(99 \mathrm{~m})$ technetium labeled annexin V. Am J Transplant 1(3):270-277

134. Van den Brande JM et al (2007) Prediction of antitumour necrosis factor clinical efficacy by real-time visualisation of apoptosis in patients with Crohn's disease. Gut 56(4):509-517

135. Blankenberg FG et al (2006) 99mTc-HYNIC-annexin V SPECT imaging of acute stroke and its response to neuroprotective therapy with anti-Fas ligand antibody. Eur J Nucl Med Mol Imaging 33(5):566-574

136. Mari $\mathrm{C}$ et al (2004) Detection of focal hypoxic-ischemic injury and neuronal stress in a rodent model of unilateral MCA occlusion/reperfusion using radiolabeled annexin V. Eur J Nucl Med Mol Imaging 31(5):733-739

137. Lampl Y et al (2006) Annexin V SPECT imaging of phosphatidylserine expression in patients with dementia. Neurology 66(8):1253-1254

138. Lorberboym $\mathrm{M}$ et al (2006) In vivo imaging of apoptosis in patients with acute stroke: correlation with blood-brain barrier permeability. Brain Res 1103(1):13-19

139. Schmitz-Valckenberg $S$ et al (2008) Real-time in vivo imaging of retinal cell apoptosis after laser exposure. Invest Ophthalmol Vis Sci 49(6):2773-2780

140. Cordeiro $M$ et al (2010) Imaging multiple phases of neurodegeneration: a novel approach to assessing cell death in vivo. Cell Death Dis 1:e3. doi:10.1038/cddis.2009.3

141. Post AM et al (2002) Imaging cell death with radiolabeled annexin $\mathrm{V}$ in an experimental model of rheumatoid arthritis. J Nucl Med 43(10):1359-1365

142. Medarova $Z$ et al (2005) Imaging beta-cell death with a nearinfrared probe. Diabetes 54(6):1780-1788 